Thorax (1968), 23, 519.

\title{
Treatment of bronchopulmonary aspergillosis with observations on the use of natamycin
}

\author{
A. H. HENDERSON AND J.E. G . PEARSON \\ From the United Bristol Hospitals, Bristol 2
}

\begin{abstract}
The problems of treatment in bronchopulmonary aspergillosis are discussed and illustrated by 11 case reports - four with aspergilloma, six with allergic aspergillosis, and two with probable allergic aspergillosis. A simple and effective treatment of aspergilloma, by local surgical evacuation of the cavity followed by irrigation with natamycin (Pimaricin), is described in three cases. Prior treatment of these cases with inhalations or intrabronchial instillations of natamycin was of doubtful benefit. The therapy of allergic aspergillosis is reviewed and an active approach is urged, based on the probable pathogenesis of the disease. This includes consideration of physical methods of removing bronchial plugs, mucolytic agents, steroids, and antifungal drugs given by aerosol in the absence of any that are suitable for systemic use. Steroids improved ventilatory function and were effective in resolving and preventing further episodes of pulmonary eosinophilia, but not usually in clearing the fungus from the bronchial tree, probably because permanent damage had already been sustained; they also carry the theoretical risk of allowing fungal invasion. Natamycin inhalations alone are considered of little value in the active disease, although their place in prophylaxis and when used with steroids remains to be evaluated. Mucolytic agents may help to clear the bronchial secretions, especially when these have become chronically impacted.
\end{abstract}

The management of the various types of bronchopulmonary aspergillosis will remain difficult until an antifungal drug satisfactory for systemic use becomes available. Our methods are discussed here with reference to 11 selected case reports.

\section{CASE REPORTS}

Cases 1 to 4 had aspergillomas; cases 5 to 11 had allergic aspergillosis, unconfirmed in cases 3 and 10 . Cases 3 and 5 to 11 represent respectively cases numbered $32,14,8,9,4,1,24$, and 3 in the previously reported series (Henderson, 1968).

CASE 1 A 66-year-old woman with a right upper lobe aspergilloma was treated by irrigation of the cavity with natamycin (Pimaricin). A suspension of $50 \mathrm{mg}$. natamycin in $8 \mathrm{ml} .45 \%$ sodium diatrizoate (Hypaque) was instilled under radiological control once a week for three months. Haemoptysis continued and there was no radiological change. The aspergilloma was then removed from its cavity through a small high axillary thoracotomy under local anaesthesia and a drainage tube was left in place. The cavity was irrigated daily for seven weeks with an aqueous suspension of natamycin of similar concentration. There

1 Present address - Peter Bent Brigham Hospital, Boston, Mass., U.S.A. were no post-operative complications. Aspergillus fumigatus was cultured from the fragments of the aspergilloma. Eighteen months later the cavity remained and strong precipitins were still present but haemoptysis had not recurred and the sputum no longer contained fungus.

CASE 2 A 45-year-old woman with a middle lobe aspergilloma suffered from repeated haemoptysis. A right upper lobectomy had previously been performed for tuberculosis. Further resection was contraindicated because of severe myasthenia gravis. She was treated with natamycin inhalations: $2 \mathrm{ml}$. of $2.5 \%$ natamycin in Alevaire was administered through an Oxygenaire nebulizer for half an hour daily for eight months. One year later the aspergilloma appeared smaller and haemoptysis had not recurred; the sputum still contained the fungus and strong precipitins persisted. The cavity was then drained locally and irrigated with natamycin as in case 1 . There were no complications. One year later haemoptysis had not recurred and the sputum no longer contained the fungus.

CASE 3 In 1959, a 50-year-old woman who had had intermittent asthma since the age of 14 years developed severe constant airways obstruction and productive cough. This followed an illness during which the head of her bed had been placed near a hole drilled in the wooden walls of the house to 
extricate baby birds which had fallen from the eaves, together doubtless with nesting debris which would have provided a good habitat for $A$. fumigatus. Four years later radiographs showed bilateral upper lobe fibrotic contraction, a large cavity at the left apex, and a small one at the right, considered to be bullous in origin. Repeated examination of sputum showed no tubercle bacilli. Tomography the following year demonstrated a small left upper lobe aspergilloma (Fig. 1a). Two years later this had greatly increased in size and another small aspergilloma had developed at the right apex. During this time she received three short courses of steroids. There was slow clinical deterioration. Sputum was repeatedly shown to contain $A$. fumigatus; strong precipitins and blood eosinophilia were present, and a skin test was positive. Prednisone and daily inhalations of natamycin in Alevaire were started in August 1966. These resulted in prompt clinical improvement, with reduction of airways obstruction and regression of the eosinophilia, though she still experienced occasional haemoptyses. In August 1967 the cavity was drained as in cases 1 and 2. The large aspergilloma (Fig. 1.b) was removed piecemeal from the left apex, and the cavity was irrigated daily for eight weeks with natamycin through the tube. Within a week she was no longer troubled by her usual productive cough, and chest radiographs showed that the left apical cavity was empty. All 140 cultures taken from various parts of the removed aspergilloma were positive for $A$. fumigatus.

CASE 4 A 27-year-old farmer was shown to have a right upper lobe mycetoma three years after a course of chemotherapy for suspected tuberculous infiltration. Sputum contained $A$. fumigatus, and strong serum precipitins were present. Segmental resection was performed. The cavity was found to be empty; no

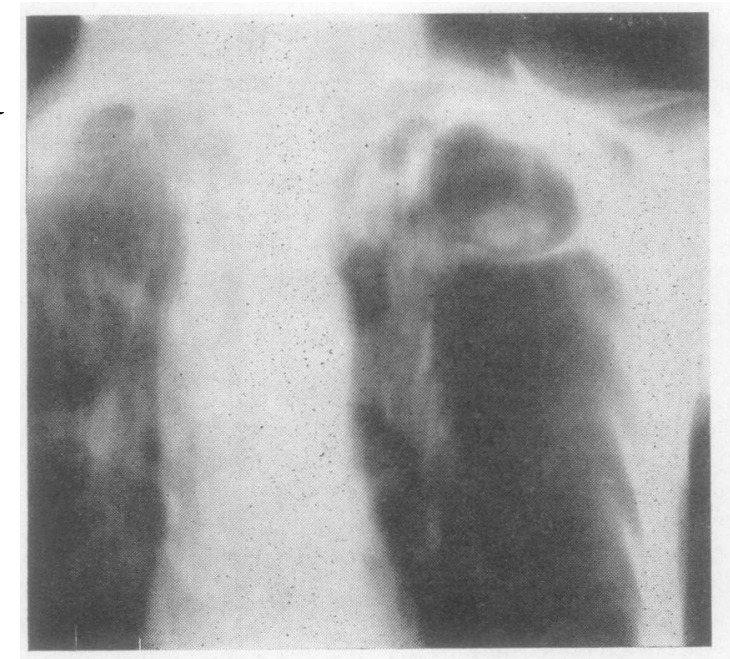

(a)

FI. 1. Case 3. Tomograms (a) at time of diagnosis of aspergilloma; (b) three years later. 
FIG. 2. Case 5.

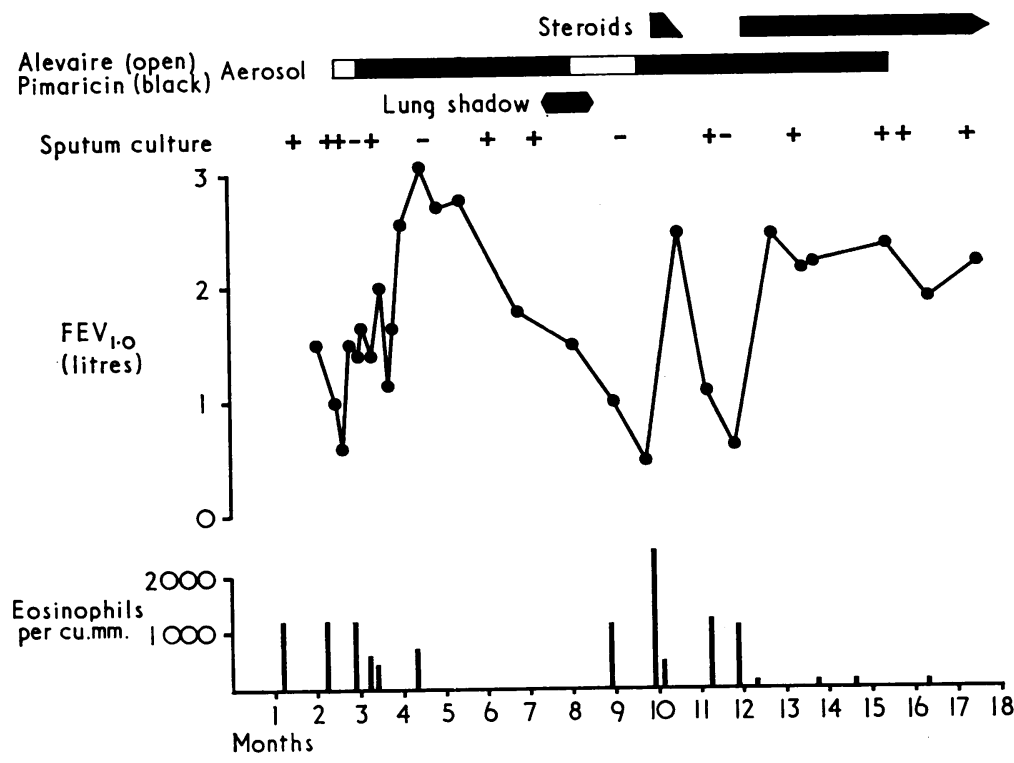

mucoid impaction persisted despite intensive physiotherapy but finally cleared with inhalations of acetyl cysteine.

CASE 7 A 40-year-old man with long-established allergic aspergillosis had been on steroid therapy for seven years, during which there had been no further episodes of pulmonary eosinophilia. He now has fixed airways obstruction, chronic upper lobe contraction, and $\boldsymbol{A}$. fumigatus persistently recoverable from his sputum. Treatment with natamycin inhalations daily for two months resulted in no improvement in ventilatory function, and the sputum remained positive.

CASE 8 A 24-year-old woman with lifelong asthma was shown to have allergic aspergillosis seven years ago. The radiological opacities then present cleared with steroids and her asthma has since been well controlled on a daily maintenance dose of 7.5 to $10 \mathrm{mg}$. prednisone. Two years later a chest radiograph showed a recurrence of left upper lobe shadowing but without any associated exacerbation of respiratory symptoms. This cleared when the dose of prednisone was temporarily increased.

CASE 9 A 20-year-old man with lifelong asthma was admitted to hospital in status asthmaticus and with a temperature of $104 \cdot 6^{\circ}$ F. An apical shadow, eosinophilia, and weak precipitins were present, the skin test was positive, and the sputum yielded Staphylococcus aureus and $A$. fumigatus. He was treated with steroids and antibiotics. The fever resolved in eight hours and the shadow a few days later. It is possible that bacterial infection developed distal to bronchial occlusion, but this case illustrates the difficulty sometimes encountered in determining whether or not an episode of pulmonary shadowing in a patient who fulfils the other criteria of allergic aspergillosis represents a specific reaction to $A$. fumigatus.

CASE 10 A 32-year-old woman with lifelong asthma had experienced episodes of pulmonary eosinophilia before starting steroid therapy eight years ago, but she was not at that time investigated for aspergillosis. She then suffered only occasional mild asthma until she was admitted to hospital with a severe attack from which she died. The skin test and precipitins were positive but no $A$. fumigatus was found in the sputum or at necropsy in the lungs. It is probable that she had allergic aspergillosis but without significant permanent damage to the bronchi, and that the fungus had cleared from the lungs during the intervening period of good ventilatory function while on steroids.

CASE 11 A 23-year-old woman, subject to intermittent astinma all her life, was recently shown to have allergic aspergillosis. With physiotherapy but no other treatment she became symptom-free and the shadows resolved.

\section{DISCUSSION}

When the fungus is localized in an aspergilloma surgical resection is the acknowledged method of treatment (Pecora and Toll, 1960), although this operation has carried a considerable morbidity (Belcher and Plummer, 1960; Robinson and McPherson, 1962). Active intervention is often unnecessary but is indicated when there is troublesome haemoptysis, diagnostic doubt, or lowered 
resistance which might allow fungal invasion (Seabury and Samuels, 1963). An aspergilloma cavity occasionally empties spontaneously, presumably by expectoration of its contents as in case 4 . In three of the present cases (cases 1,2, and 3) the aspergilloma was treated by the minor surgical procedure of evacuating the cavity through a small local thoracotomy (Loeckell, 1964). This was covered by the antifungal agent natamycin, given by inhalation or intrabronchial instillation preoperatively and then by direct irrigation of the cavity. The procedure proved simple, effective, free from complications, and practicable when more extensive surgery was contraindicated.

The treatment of allergic aspergillosis is more difficult to evaluate. The natural history of the condition is incompletely understood, its relative contribution to the overall clinical picture often uncertain, and its more obvious manifestations intermittent. A rational approach to therapy may provisionally be based on a working conception of its pathogenesis (Henderson, 1968). The severity of the local bronchial reactions which lead to airways obstruction, bronchial plugging, and subsequently to permanent bronchial dilatation probably reflects a vicious circle where growth of the inhaled fungus, trapped in the viscid secretions its presence provoked, continues to provide an increasing quantity of antigenic material. The aim of treatment should therefore be to break this vicious circle, by suppression of the allergic response and by removal of the antigens. Even when symptoms are minimal because of the localized nature of the disease, early and strenuous treatment is probably important to prevent lung damage.

Atopic subjects should avoid obvious potentially rich sources of the fungal spores, such as rotting plant material, although they will be unlikely to escape exposure entirely in Britain. Probable regional differences in atmospheric spore concentrations may also prove relevant to prevention of the disease. Specific hyposensitization has been shown to reduce bronchial hypersensitivity but is not altogether free from risk (Citron and Pepys, 1958 ; Pepys, 1966) and its place in prophylaxis is difficult to assess.

In treatment of the active disease steroids have been widely used to suppress the allergic reaction. In adequate dosage they relieved airways obstruction, resolved current lung shadows, and prevented further episodes of pulmonary eosinophilia in all 11 cases so treated in the previously reported series (Henderson, 1968) (cases 5, 7, 8, 9, and 10). Sputum remained positive in all these cases except in case 10 . It is probably difficult to clear a damaged bronchial tree of fungus, whereas if permanent

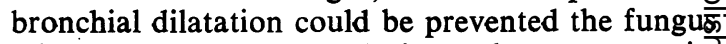
might be expectorated during subsequent remis sions of the allergic condition. Chronic upper lobe्d contraction is seen in some long-standing cases o allergic aspergillosis, and in one such patient op̣ steroid therapy the fungus was found at necropsi to have invaded the lung parenchyma wity granuloma formation. The risk of fungal invasio when host resistance is lowered by small doses of steroids is, however, probably insignificant unles $\xi^{\circ}$ there is heavy fungal colonization of previousl $f$ damaged lung. Further understanding of the pos? sible immunological changes underlying the teno dency for the recurrent episodes of pulmonary eosinophilia to die out naturally after a numbees of years may lead to more specific treatment.

The condition has also been treated with $\Phi$ variety of antifungal agents, given by aerosol o용 intrabronchial instillation, since none suitable for systemic use is yet available. Some success has more recently been reported with natamycio (Edwards and La Touche, 1964 ; Fraser, 1965). İ the present series natamycin inhalations were associated with some initial improvement in cases 5 and 6 , but both patients relapsed despite cono tinued treatment, and the sputum still contained the fungus (Fig. 2). Antifungal agents given bo inhalation will not easily penetrate the viscid secre? tions of allergic aspergillosis or into areas of lung damage (case 7). Their place in conjunction wit other methods of treatment and in prophylaxis remains to be established.

Removal of the viscid secretions remains separate problem, especially when these ar chronically impacted in damaged bronchi. Physio therapy may prove adequate where this is littlo or no permanent distortion of the bronchi (case 11); bronchial lavage and bronchoscopic suctiog have been effectively used (Ellis, 1965) ; and pre liminary experience with the mucolytic agent acetyl cysteine, suggests that this approach may be helpful (case 6). In addition, antibiotics are somes times indicated for bacterial infection distal to bronchial occlusion (case 9) (Plummer, 1958).

Invasive aspergillosis has rarely been diagnoseg in life but may be recognized more frequently with the aid of screening tests for precipitins (Hende? son, English, and Vecht, 1968). Amphotericin 8 has been used in a few cases (Newcomer, Sternberg, Wright, and Reisner, 1959; Peer, $1960_{0}^{\circ}$ Conen, Walker, Turner, and Field, 1962 ; Utz an Andriole, 1962) and despite its toxicity it has beef recommended in the treatment of this condition (American Thoracic Society Committee of Therapy, 1963). 
We are most grateful to Mr. R. H. Belsey, Dr. D. R. Coles, Mr. J. L. Griffith, Dr. D. Mattingly, and Dr. D. B. Shaw for permission to study and to present details of patients under their care; and to Messrs. Mycofarm-Delft, of Holland, who generously provided the Pimaricin.

\section{REFERENCES}

American Thoracic Society Committee on Therapy (1963). Fungal infections of the lungs. Amer. Rev. resp. Dis., 87, 784.

Belcher, J. R., and Plummer, N. S. (1960). Surgery in bronchopulmonary aspergillosis. Brit. J. Dis. Chest, 54, 335.

Citron, K., and Pepys, J. (1958). In Fungous Diseases and their Treatment, ed. Riddell, R. W., and Stewart, G. T., p. 134. Butterworth, London.

Conen, P. E., Walker, G. R., Turner, J. A., and Field, P. (1962). Invasive primary aspergillosis of the lung with cerebral metastasis and complete recovery. Dis. Chest, 42, 88 .

Edwards, G., and La Touche, C. J. P. (1964). The treatment of bronchopulmonary mycoses with a new antibiotic pimaricin. Lancet, 1, 1349.

Ellis, R. H. (1965). Total collapse of the lung in aspergillosis. Thorax, 20, 118 .
Fraser, J. W. (1965). Aspergilloma following pulmonary tuberculosis. Scot. med. J., 10, 318.

Henderson, A. H. (1968). Allergic aspergillosis: review of 32 cases. Thorax, 23, 501.

—— English, M. P., and Vecht, R. J. (1968). Pulmonary aspergillosis: a survey of its occurrence in patients with chronic lung disease and a discussion of the significance of diagnostic tests. Thorax, 23,513 .

Loeckell, H. (1964). Über die transthorakale Austräumung eines Lungenmyzetoms mittels Maurerdrainage. Prax. Pneumol., 18, 757.

Newcomer, V. D., Sternberg, T. H., Wright, E. T., and Reisner, R. M. (1959). Current status of amphotericin B in the treatment of the systemic fungus infections. J. chron. Dis., 9, 353.

Pecora, D. V., and Toll, M. W. (1960). Pulmonary resection for localized aspergillosis. New Engl. J. Med., 263, 785.

Peer, E. T. (1960). Case of aspergillosis treated with amphotericin 'B'. Dis. Chest, 38, 222.

Pepys, J. (1966). Pulmonary hypersensitivity disease due to inhaled organic antigens. Postgrad. med. J., 42, 698.

Plummer, N. S. (1958). In Fungous Diseases and their Treatment, ed. Riddell, R. W., and Stewart, G. J., p. 234. Butterworth, London.

Robinson, C. L. N., and McPherson, A. R. (1962). Bronchopulmonary aspergilloma. Canad. J. Surg., 5,411 .

Seabury, J. H., and Samuels, M. (1963). The pathogenetic spectrum of aspergillosis. Amer. J. clin. Path., 40, 21.

Utz, J. P., and Andriole, V. T, (1962). Unpublished data, cited by Andriole, V. T., and Kravetz, H. M. (1962). The use of amphotericin B in man. J. Amer. med. Ass., 180, 269. 